\title{
A formulation of the linear discrete Coulomb friction problem via convex optimization
}

\author{
Vincent Acary, Florent Cadoux, Claude Lemaréchal, and Jérôme Malick \\ Inria Rhône-Alpes, 655 avenue de l'Europe, 38330 Montbonnot, France
}

\begin{abstract}
Key words nonsmooth mechanics, contact mechanics, Coulomb friction, Painlevé's problem, fixed-point theorem, convex analysis, convex optimization, second-order cone programming

MSC (2000) 49J52, 70F40, 90C 25

This paper presents a new formulation of the dynamical Coulomb friction problem in finite dimension with discretized time. The novelty of our approach is to capture and treat directly the friction model as a parametric quadratic optimization problem with second-order cone constraints coupled with a fixed point equation. This intrinsic formulation allows a simple existence proof under reasonable assumptions, as well as a variety of solution algorithms. We study mechanical interpretations of these assumptions, showing in particular that they are actually necessary and sufficient for a basic example similar to the so-called "paradox of Painlevé". Finally, we present some implementations and experiments to illustrate the practical aspect of our work.
\end{abstract}

Copyright line will be provided by the publisher

\section{Introduction, motivations}

We consider the problem of simulating the dynamics of mechanical systems which involve unilateral contact between their parts or with external objects. Such systems are common in engineering applications and they have been extensively studied by the community of contacts mechanics: see for instance [Wri06, WL08] in the context of computational contact mechanics for solids and structures, [Mor94, RJMR96, ZVJL00] for applications to granular materials, divided media and powders materials, [FM04] for applications in civil engineering, [PG96, Aba00, TLG ${ }^{+}$08, GCSL09] for multibody systems and robotics, and finally [Bar93] for computer graphics. When, in addition to unilateral constraint, Coulomb's friction occurs at contact points, the resulting problem to be solved at each time-step (that we call the incremental problem) becomes so difficult that, up to our knowledge, no existing algorithm is able to solve it (when a solution exists) with guaranteed convergence. In this paper, we focus on a convex-analysis reformulation of the incremental problem that opens the way to (i) a proof of existence of a solution to the problem; and (ii) a numerical procedure to solve it.

Existence of solutions to the discrete friction problem. In addition to the theoretical interest, the existence question is crucial also in practice. Indeed, it may happen that the algorithms dedicated to solving the incremental problem fail, but it is often not obvious whether the failure occurred because no solution exists (meaning that we should probably reconsider the model) or because our algorithm just did not find it (in which case, we should try to improve the algorithm). In both cases, finding out whether a solution exists or not is important both from the theoretical and the practical viewpoints.

Existence of solutions to problems involving a mechanical system with Coulomb's friction has been addressed in many papers under a wide range of assumptions. For a recent and comprehensive presentation of the standard formulations of the frictional contact static, quasi-static and dynamic problems of linear elastic continuum media, we refer to [BH09]. In the seminal work [DL72], the existence and uniqueness of a solution is shown for the static problem with bilateral contact and Tresca's friction (constant friction threshold independent on the normal force). The term static refers to the fact that the friction is written in terms of position rather than velocities. Although this problem has a poor physical significance, it was the starting point of several extensions toward more realistic situations. The first existence result for the static Coulomb problem with unilateral contact is due to [NJH80] for a two-dimensional elastic strip, and it was generalized by [Jar83, Jar84] to more general two-dimensional problems. A recent account on existence and uniqueness problems for continuum media can be found in [EJK05, HS02]. Briefly speaking, existence is obtained by a fixed-point argument on the friction threshold providing that the coefficient of friction is small enough. In this quasi-static case, Coulomb's law is written in a more realistic setting in terms of velocity but the inertia effects are neglected. The existence problem for the quasi-static case has been addressed by [And00, RC01] by calling for a time-discretization technique in order to obtain an incremental problem which appears as a particular static one. The equilibrium problem resulting from the quasi-static and 
dynamic problems has also been investigated in [BH09]. In this latter work, sufficient conditions ensuring an infinity of equilibrium configurations for arbitrarily small coefficients of friction are given. As far as we know, the dynamic problem for continuum media with unilateral contact and Coulomb's friction is still an open issue, even if some attempts have been made in [JE99, EJ03] with normal compliance models.

In the context of finite-dimensional systems (rigid-body systems or space-discretized systems by finite-element methods), existence and uniqueness has been obtained in [Bal00] for the dynamics with perfect unilateral constraints, mainly under an analyticity assumption on the data. When the Coulomb friction is involved, many authors have investigated static, quasi-static or evolution problems and its dramatic features, such as non-existence and non-uniqueness, which have been known for a long time. Among others, see [GB99] about the so-called "paradox of Painlevé", which is the most famous example of such systems for which non-existence may occur. Nevertheless, some results can be cited such as [BB05], where some decoupling in the stiffness matrix is assumed. In the static case, which is the static counterpart of the problem of Duvaut-Lions, Janovský [Jan81] and Haslinger [Has83, Has84] prove existence of a solution in the two-dimensional case with scleronomous boundary conditions, for any coefficient of friction. This result is fact contained in the present paper. For the quasi-static case, a counter example to uniqueness is given by [Ba199] for arbitrarily small friction coefficients.

For the time-discretized problem and finite-dimensional systems, the literature seems more scattered. In [Ala97], an indirect attempt to prove existence of solutions is made by studying the convergence of the method of Alart and Curnier [AC91], dedicated to solving the incremental problem. In [Ala93], necessary and sufficient conditions are given for existence of solutions to the discrete problem, derived from the static formulation. In the two-dimensional case or for a faceted three-dimensional cone, the advocates of the linear complementarity (LCP) approach propose several existence results [ST96, APS99, Ste98], using the copositive LCP theory. The most advanced existence result appeared in [KP98], which studies the semi-coercive case (where the stiffness matrix is only positive semi-definite). Existence is proved under the assumption that the data of the problem lie in a specific cone. We will present here a similar result: we need a slightly more stringent assumption, but we propose a simpler proof using only standard convex analysis tools. Furthermore we use a formulation of the problem which opens the way to reasonably stable solution algorithms.

Note finally that the mathematical analysis of the incremental problem for the space- and time-discretized dynamics is very similar to that of a quasi-static problem for finite-dimensional situations; therefore our results as well as those of [KP98, PS99] can be applied to the dynamic case, providing that some care is taken when formulating the problem (see the discussion in [AC10, Section 3]). Conversely, the results in [ST96, APS99, Ste98] apply only for scleronomous external constraints. A straightforward application of the latter results are not possible for the time-discretization of the quasi-static problem.

Numerical resolution. A review of the main existing approaches to solve numerically the discrete incremental frictional contact problem is given in [AB08]; let us comment them briefly here. The algorithms for solving LCPs have been extensively used when the friction cone is polyhedral (two-dimensional case or cone-faceting approach). In [Kla86, KB88, AFSP91, PG96, ST96, PT96], pivoting methods such as Lemke's are used to solve the LCP. This is the only example of numerical algorithms proved to compute a solution when an existence criterion is satisfied [ST96, APS99]. For the Coulomb cone, the projection/splitting method for finite-dimensional variational inequalities [MD87, MD88, DSF91, Fen95, DSF98, JT88, JM92, JAJ98] and the nonsmooth (semi-smooth or generalized) Newton methods [CA88, AC91, CKPS98, PK94, LGW98] are the most widespread methods for solving the incremental problem. Unfortunately, there is no general proof of convergence for such methods.

Contributions and outline of the paper. The main contributions of the present paper are the following ones:

1. an optimization-based reformulation of the incremental problem;

2. an existence result for this problem (with a direct proof);

3. a discussion on the practical applicability of the existence result (we show in particular that a simple abstract reasoning gives existence in many practical situations);

4. a resolution procedure based on the proposed reformulation (illustrated with some numerical experiments).

This paper is organized as follows. We briefly recall in $\S 2$ the Coulomb friction model, together with an illustrative example where no solution can exist. Then, we give to this model a parametric-optimization formulation in $\S 3$, and we prove existence of a solution under a natural geometric assumption in $\S 4$ (and we compare it with the one of [KP98]). Section 5 discusses our assumption for existence, giving a list of cases where it is satisfied and applying it to the counterexample of $\S 2$. Finally Section 6 illustrates a possible algorithmic approach to solve the incremental problem. 
The originality of this paper is that it makes an extensive use of convex analysis, shedding new light on the problem. The nice interplay between convex analysis and mechanics (and especially contact mechanics) comes back to the seminal work of J.-J. Moreau (for instance [Mor66]). We stress here in particular the role of conic optimization ([BV04] is suggested for this topic). The notions as well as notation are standard and follow closely those of the reference textbooks on convex analysis [Roc70, HUL93].

\section{Linear, discrete-time contact mechanics with friction}

We present here the standard modeling of the multi-body friction problem, coming when discretizing the dynamics of a mechanical system involving contact and friction. The formulation ends up with the system (2.3) (with friction model (2.2)) that is the object of this paper. Details on how to produce the data $M, f, H, w$ of (2.3) for a general mechanical problem are skipped, but $\S 2.2$ provides an example (see also [Cad09b, AC10]). Note that impacts are not considered in this paper, but using Moreau's rule [Mor88] they can be easily added.

\subsection{Elements of modeling, incremental problem}

We consider a mechanical system in a $d$-dimensional space identified to $\mathbb{R}^{d}$ (in practice, $d=2$ or $d=3$ ) with a finite number $m$ of degrees of freedom. We assume the system is discretized in time, and focus on one time-step of the evolution. Unilateral contact is assumed to occur in a finite number $n$ of points in the system. Labelling arbitrarily the contacting bodies by $A^{i}$ and $B^{i}$ at the $i$-th contact point, define the unit normal vector $e^{i}$ from $B^{i}$ towards $A^{i}$, the discretized relative velocity $u^{i} \in \mathbb{R}^{d}$ of $A^{i}$ with respect to $B^{i}$ and the discretized impulse $r^{i}$ exerted by $B^{i}$ on $A^{i}$ over the current time-step. Assuming linear discretized kinematics, the generalized velocities $v \in \mathbb{R}^{m}$ are related to the relative velocities at contact points $u:=\left(u^{1}, \ldots, u^{n}\right) \in \mathbb{R}^{n d}$ and to the discretized impulses $r:=\left(r^{1}, \ldots, r^{n}\right) \in \mathbb{R}^{n d}$ by affine equations. Specifically, $(u, v, r)$ are related by the kinematic relation and dynamical equation

$$
u=H v+w, \quad M v+f=H^{\top} r
$$

where $H \in \mathbb{R}^{n d \times m}, w \in \mathbb{R}^{n d}, M \in \mathbb{R}^{m \times m}$ and $f \in \mathbb{R}^{m}$ are known.

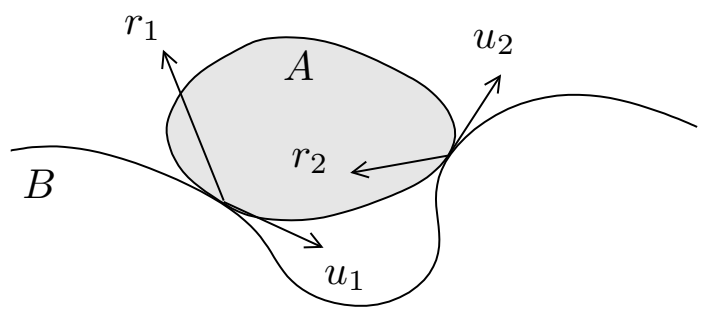

Fig. 1 Unknowns $u$ and $r$

In this paper, we always make the assumption - which is standard in that situation:

The matrix $M$ is symmetric and positive definite.

Contact at each point $i$ gives additional coupling constraints. Here, we model friction using Coulomb's law. The velocity $u^{i}$ and impulse $r^{i}$ are assumed to satisfy Coulomb's law, stating that $\left(u^{i}, r^{i}\right) \in \mathcal{C}\left(e^{i}, \mu^{i}\right)$ where the set $\mathcal{C}\left(e^{i}, \mu^{i}\right)$ is defined by a disjunctive constraint as follows. Let $\mu^{i} \in\left[0, \infty\left[\right.\right.$ and $e^{i} \in \mathbb{R}^{d}$ (standing for $\mu^{i}$ and $e^{i}$ ); a vector $z \in \mathbb{R}^{d}$ is decomposed as $z=z_{T}+z_{N}$, with $z_{T}$ orthogonal to $e^{i}$. Then the $\operatorname{set} \mathcal{C}\left(e^{i}, \mu^{i}\right) \subset \mathbb{R}^{d \times d}$ is defined by

$$
(u, r) \in \mathcal{C}\left(e^{i}, \mu^{i}\right) \Longleftrightarrow\left\{\begin{array}{ccc}
\text { either: } & r=0 \text { and } u_{N} \geqslant 0 & \text { (take off) } \\
\text { or: } & \left\|r_{T}\right\| \leqslant \mu r_{N} \text { and } u=0 \\
\text { or: } & \left\{\begin{array}{c}
0<\left\|r_{T}\right\|=\mu r_{N} \text { and } \\
\exists \alpha>0: r_{T}=-\alpha u
\end{array}\right\} & \text { (sticking) }
\end{array}\right.
$$

(note that sliding implies $u_{N}=0$ ). The take-off case occurs when the normal velocity is nonnegative and the contact force is zero, which means that there is no attractive force (no adherence, this models dry friction) nor repulsive force when the bodies separate. The sticking case occurs when the relative velocity is zero, then the contact force can lie anywhere in its cone. Finally, the sliding case occurs when the two bodies are moving tangentially to each other. In this case, the contact 
force must be "as opposed as possible" to the relative velocity (this is often called the maximum dissipation principle [Mor88]). Altogether, the incremental problem we focus on is

$$
\left\{\begin{array}{l}
M v+f=H^{\top} r \\
u=H v+w \\
\left(u^{i}, r^{i}\right) \in \mathcal{C}\left(e^{i}, \mu^{i}\right) \text { for all } i \in 1, \ldots, n
\end{array}\right.
$$

with $\mathcal{C}(\cdot, \cdot)$ defined by $(2.2)$.

The incremental problem (2.3) is an archetype and a template of the problem that we have to solve in more general situations under the static or quasi-static assumptions, or in the nonlinear mechanical case when an outer Newton procedure is performed.

\subsection{Illustration of the modeling}

In some particular instances (essentially, when $d=2$ and $n$ is small) (2.3) can be solved by hand. In this subsection, we describe a toy problem, inspired by the so-called paradox of Painlevé [Pai95], whose modeling results in such an incremental problem. In addition to its illustrative interest, it will be used later as a use-case for our existence criterion. It has only one degree of freedom and one contact, in dimension 2, and shows that problem (2.3) may have no solution (Example 2.1).

Consider the situation depicted on Figure 2.

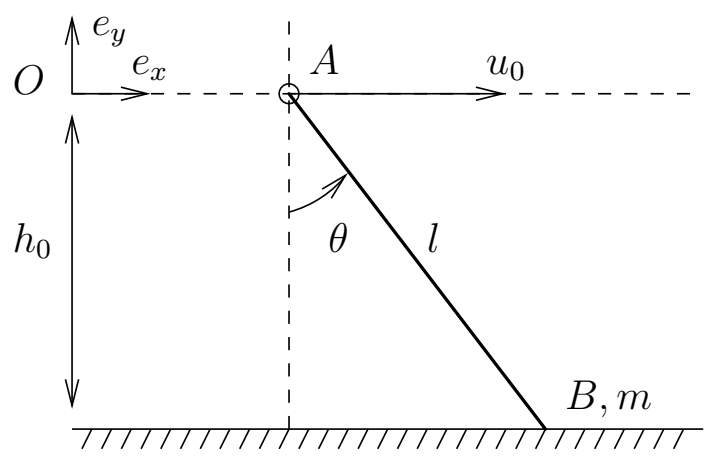

Fig. 2 A very simple contact problem

The point $A$ is moving along the axis $O x$ with fixed velocity $u_{0}$ (possibly, $u_{0}<0$, in which case the point $A$ is moving leftwards). A rigid rod of length $l$ holding a mass $m$ at its lower end $B$ is articulated with $A$ by a perfect pivot joint. The end $B$ of the bar is subject to unilateral contact with the ground : it can either touch the ground as on Figure 2, or take off. In case of contact, the ground applies a force (or impulse, to allow for impacts) $\lambda$ onto the bar at $B$. The only degree of freedom of this system is parametrized by the angle $\theta$, and it is subject to the gravity field $g$ along $O y$ (with $g>0$ meaning that the gravity is directed upwards, and $g<0$ that it is directed downwards).

The evolution of the system is governed by the equation

$$
m l^{2} \ddot{\theta}=m g l \sin (\theta)+l\left(\cos (\theta) \lambda_{x}+\sin (\theta) \lambda_{y}\right),
$$

and its discretization yields (see [AC10]) the incremental problem (2.3) with data

$$
M=m l^{2}, f=-m g l h \sin \theta-m l^{2} v_{0}, H=l\left[\begin{array}{c}
\cos \theta \\
\sin \theta
\end{array}\right], w=\left[\begin{array}{c}
u_{0} \\
0
\end{array}\right] .
$$

Example 2.1 (Necessary and sufficient conditions for existence) Take the values in (2.5) : $m=1, l=1, g=-1$, $h=1$ and $v_{0}=0$. We do not fix the value of $u_{0}, \mu$ and $h_{0}$ at the moment, and assume that $h_{0}<l$ so that the contact can be active, with $\theta \in] 0, \pi / 2[$. It is easy to prove [AC10] that problem (2.3) has a solution if and only if

$$
u_{0} \leqslant 0 \quad \text { or } \quad\left[u_{0}>0 \text { and } \tan \theta>\mu\right] .
$$

In particular, this gives a simple example where no solution exists.

This is coherent with intuition: when $\tan \theta>\mu$, the torque applied by the friction force $r$ acts on the bar counterclockwise, and allows to compensate the effect of gravity which tends to drive the bar downwards, towards the ground. If 
$\tan \theta=\mu$, the friction force exerts no torque at all and plays no role. Finally, if $\tan \theta<\mu$, the torque applied by the friction force $r$ acts clockwise and increases the effect of gravity by driving $B$ towards the ground as well. The friction force being unable to compensate gravity, nothing prevents the bar from penetrating the ground and the unilateral constraint has to be violated, therefore no physical solution exists.

\section{Conic optimization formulation}

In this section, we reformulate the incremental problem (2.3) as a parametric conic optimization problem coupled with a fixed-point equation. This formulation lends itself to a simple existence result $(\S 4)$ and to a variety of algorithmic approaches, mentioned in $\S 6$.

\subsection{Notation and recalls of convex analysis}

We fix our notation and recall some concepts of convex analysis that will be used throughout this paper.

We work in finite dimension spaces identified to $\mathbb{R}^{k}$ for various $k$, equipped with the canonical Euclidean norm denoted by $\|\cdot\|$. Given a vector $x \in \mathbb{R}^{k}$, the subscripts " $N$ " and " $T$ " indicate normal and tangential components of a vector with respect to a given unit vector $e \in \mathbb{R}^{k}$. In other words,

$$
x_{N}:=x^{\top} e \in \mathbb{R} \quad \text { and } \quad x_{T}:=x-x_{N} e \in \mathbb{R}^{k} .
$$

A subset $S$ of $\mathbb{R}^{k}$ is called a cone if $\alpha x \in S$ for any $x \in S$ and $\alpha>0$. Note that $0 \in S$ if $S$ is a closed cone. The basic object of our study is the so-called second-order cone $K_{e, \mu}$, defined in terms of a direction $e \in \mathbb{R}^{k}$ and a parameter (the friction coefficient) $\mu \in] 0,+\infty[$ by

$$
K_{e, \mu}:=\left\{x \in \mathbb{R}^{k}:\left\|x_{T}\right\| \leqslant \mu x_{N}\right\}
$$

We also consider the two extreme cases

$$
K_{e, 0}:=\left\{x \in \mathbb{R}^{k}: x_{T}=0, x_{N} \geqslant 0\right\} \quad \text { and } \quad K_{e, \infty}:=\left\{x \in \mathbb{R}^{k}: x_{N} \geqslant 0\right\} .
$$

In the sequel, $\mu$ will represent a friction coefficient and it will lie in $[0,+\infty]$. The closed convex cone $K_{e, \mu}$ is also called ice-cream, Lorentz or Coulomb cone, and has received a lot of interest from the optimization community as an important instance in conic programming (see for instance the review [AG03] or references in the textbook [BV04]). In our context, it appears naturally when expressing Coulomb's friction law, as explained in forthcoming Lemma 3.1. Note also that products of second-order cones in a Cartesian product space still form closed convex cones.

The normal cone to $S$ at a point $\bar{s} \in S$ is the closed convex cone defined by

$$
N_{S}(\bar{s}):=\left\{x \in \mathbb{R}^{k}:(s-\bar{s})^{\top} x \leqslant 0, \text { for all } s \in S\right\}
$$

Normal cones generalize in convex geometry orthogonal subspaces in linear geometry: if $V$ is a vector space, then for any $v \in V, N_{V}(v)=V^{\perp}$. The dual cone of $S$ is the closed convex cone of $\mathbb{R}^{k}$ defined by

$$
S^{*}:=\left\{x \in \mathbb{R}^{k}: s^{\top} x \geqslant 0, \text { for all } s \in S\right\} .
$$

If $S$ is a closed convex cone, we see on definitions that $S^{*}=-N_{S}(0)$ and moreover there holds (see [HUL93, Ex. III.5.2.6])

$$
N_{S}(\bar{s})=-S^{*} \cap \bar{s}^{\perp}
$$

in addition, $\left(S^{*}\right)^{*}=S$. It is easy to see that the dual of the second-order cone $K_{e, \mu}$ (with $\left.\mu \in\right] 0, \infty[$ ) is also a second-order cone:

$$
K_{e, \mu}^{*}=K_{e, \frac{1}{\mu}}
$$

This also holds for $\mu=0$ and $\mu=\infty$, with the convention that $1 / 0=\infty$ and $1 / \infty=0$ : we have indeed

$$
\left(K_{e, 0}\right)^{*}=K_{e, \infty} \quad \text { and } \quad\left(K_{e, \infty}\right)^{*}=K_{e, 0} .
$$

Note finally that the interior of $K_{e, \mu}$ has an explicit expression: for $\left.\mu \in\right] 0, \infty$, we have

$$
\operatorname{int} K_{e, \mu}=\left\{x \in \mathbb{R}^{k}:\left\|x_{T}\right\|<\mu x_{N}\right\} \text {. }
$$


A convex function is not necessarily differentiable everywhere, and gradients of $f$ at $x$ generalize to subgradients: those $g$ satisfying

$$
f(y) \geqslant f(x)+g^{\top}(y-x), \quad \text { for all } y \in \mathbb{R}^{k} .
$$

The set of subgradients of $f$ at $x$ is a closed and convex set, denoted by $\partial f(x)$ and called the subdifferential of $f$ at $x$. For example, consider the indicator function $\mathrm{i}_{S}$ of the convex set $S$, which takes the value 0 on $S$ and $+\infty$ outside: this is indeed a convex function, whose domain is $S$. Its subdifferential is easy to compute:

$$
\partial \mathrm{i}_{S}(x)=N_{S}(x)
$$

Note finally that a minimum $x$ of $f$ is characterized by the optimality condition $\partial f(x)=0$, generalizing the condition $\nabla f(x)=0$ when $f$ is smooth (this is easy to see on the definition (3.5)).

\subsection{Conic complementarity constraints}

Operating a change of variables inspired by the so-called bipotential [DSF98], we introduce $\tilde{u} \in \mathbb{R}^{n d}$ defined by

$$
\tilde{u}^{i}:=u^{i}+\mu^{i}\left\|u_{T}^{i}\right\| e^{i} \quad \text { for } i=1, \ldots, n .
$$

If $\mu^{i}=0$, this change has no impact; so we treat the two cases $\left(\mu_{i}=0\right.$ and $\left.\mu_{i} \neq 0\right)$ separately and we consider

$$
I:=\left\{i \in\{1, \ldots, n\}: \mu^{i} \neq 0\right\} \quad \text { and } \quad n_{I}:=\operatorname{Card} I .
$$

For $i \in I$, the change $u^{i} \rightarrow \tilde{u}^{i}$ can be written with the help of a new variable $s^{i}$ :

$$
s^{i}:=\left\|u_{T}^{i}\right\|=\left\|\tilde{u}_{T}^{i}\right\| \quad \text { and } \quad \tilde{u}^{i}=u^{i}+\mu^{i} s^{i} e^{i}
$$

Now we introduce a matrix $E \in \mathbb{R}^{n d \times n_{I}}$ to write the change synthetically: $E$ is constructed by concatenating $n_{I}$ columns $E_{i} \in \mathbb{R}^{n d}$, where $E_{i}$ is itself the concatenation of $n$ vectors of $\mathbb{R}^{d}$, all zero except for the $i^{\text {th }}$ which is $\mu^{i} e^{i}$. An example to fix ideas for $d=2, n=3$ is

$$
e^{1}=e^{2}=e^{3}=\left[\begin{array}{l}
0 \\
1
\end{array}\right], \quad \mu^{1}=1, \mu^{2}=0, \mu^{3}=2 ; \quad \text { then } E=\left[\begin{array}{cc}
0 & 0 \\
1 & 0 \\
0 & 0 \\
0 & 0 \\
0 & 0 \\
0 & 2
\end{array}\right] \text {. }
$$

The following observation will be useful: for any $s$ and $t$ in $\mathbb{R}^{n_{I}}$ and $i=1, \ldots, n$,

$$
(H v+w+E t)^{i}= \begin{cases}(H v+w+E s)^{i} & \text { if } i \notin I \\ (H v+w+E s)^{i}+\mu^{i}\left(t^{i}-s^{i}\right) e^{i} & \text { if } i \in I .\end{cases}
$$

In terms of the new variables $\tilde{u}:=\left(\tilde{u}^{1}, \ldots, \tilde{u}^{n}\right) \in \mathbb{R}^{n d}$ and $s:=\left\{s^{i}: i \in I\right\} \in \mathbb{R}^{n_{I}}$, we thus write (3.7) simply as

$$
\tilde{u}=u+E s
$$

and we proceed to reformulate (2.3) with $\tilde{u}$ and $s$. First, the kinematic equation in (2.1) is

$$
\tilde{u}=H v+w+E s .
$$

Second, the impulse-velocity formulation (2.2) of the friction is equivalent to $n$ conic complementarity systems:

Lemma 3.1 (Reformulation of Coulomb's law) The couple $\left(u^{i}, r^{i}\right)$ satisfies Coulomb's law (2.2) if and only if the couple $\left(\tilde{u}^{i}, r^{i}\right)$ - with $\tilde{u}^{i}$ defined by (3.7) - satisfies

$$
\left(K_{e^{i}, \mu^{i}}\right)^{*} \ni \tilde{u}^{i} \perp r^{i} \in K_{e^{i}, \mu^{i}} .
$$


Pro of. To alleviate notation and fit with (2.2), we omit super- and subscripts.

If (2.2) is valid, then $r \in K$ and $u_{N} \geqslant 0$, so that $\tilde{u} \in K^{*}$; it suffices to check that $\tilde{u} \perp r$. In the take-off case $(r=0)$ and for sticking ( $u=0$ so that $\tilde{u}=0)$ it is obvious, and in the sliding case we have

$$
\begin{aligned}
\tilde{u} \cdot r & =u_{T} \cdot r_{T}+r_{N}\left(\mu\left\|u_{T}\right\|\right) \\
& =-\left\|r_{T}\right\|\left\|u_{T}\right\|+r_{N}\left(\mu\left\|u_{T}\right\|\right) \\
& =-\mu\left\|r_{N}\right\|\left\|u_{T}\right\|+r_{N}\left(\mu\left\|u_{T}\right\|\right)=0 .
\end{aligned}
$$

In each case, (3.10) is satisfied.

Conversely, if (3.10) is valid, we see that $u_{N}+\mu\left\|u_{T}\right\|=\tilde{u}_{N} \geqslant \mu\left\|\tilde{u}_{T}\right\|=\mu u_{T}$ (since $\tilde{u} \in K^{*}$ ) so that $u_{N} \geqslant 0$. Consider three cases:

(i) If $r=0$ then, as $u_{N} \geqslant 0,(2.2)$ is satisfied (take-off case).

(ii) If $\tilde{u}=0$ then $u=0$ and since $r \in K$ by assumption, (2.2) is satisfied (sticking).

(iii) If $\tilde{u} \neq 0$ and $r \neq 0$, then $\tilde{u} \in \partial K^{*}$, so that $u_{N}=0$, and $r \in \partial K \backslash\{0\}$. Moreover, $0=\tilde{u}^{\top} r=u_{T}^{\top} r_{T}+\mu r_{N}\left\|u_{T}\right\|$ so that $\mu r_{N}\left\|u_{T}\right\|=\left|u_{T}^{\top} r_{T}\right| \leqslant\left\|u_{T}\right\|\left\|r_{T}\right\|=\left\|u_{T}\right\| \mu r_{N}$. The Cauchy-Schwarz inequality is an equality, which proves that $u_{T}$ and $r_{T}$ are collinear. Finally, since $u_{T}^{\top} r_{T}=-\mu r_{N}\left\|u_{T}\right\|<0$, these vectors have opposite directions and (2.2) is satisfied (sliding case).

In each case, (2.2) il satisfied.

Along with (3.10), we introduce the product-cone

$$
L:=K_{e^{1}, \mu^{1}} \times \cdots \times K_{e^{n}, \mu^{n}} \subset \mathbb{R}^{n d},
$$

so that the conic complementary constraints (3.10) can be condensed in a single one

$$
L^{*} \ni \tilde{u} \perp r \in L .
$$

Note that the dual cone of $L$ is

$$
L^{*}=K_{e^{1}, \mu^{1}}^{*} \times \cdots \times K_{e^{n}, \mu^{n}}^{*}=K_{e^{1}, \frac{1}{\mu^{1}}} \times \cdots \times K_{e^{n}, \frac{1}{\mu^{n}}} .
$$

In summary, the incremental problem (2.3) is thus equivalent to the following conic complementarity constraint

$$
\left\{\begin{array}{l}
M v+f=H^{\top} r \\
\tilde{u}=H v+w+E s \\
L^{*} \ni \tilde{u} \perp r \in L \\
s^{i}=\left\|\tilde{u}_{T}^{i}\right\|, \quad \text { for } i \in I,
\end{array}\right.
$$

where the variables are $(v, r, \tilde{u}, s) \in \mathbb{R}^{m} \times \mathbb{R}^{n d} \times \mathbb{R}^{n d} \times \mathbb{R}^{n_{I}}$.

The idea will be to extract from (3.13) the conic complementarity problem

$$
\left\{\begin{array}{l}
M v+f=H^{\top} r \\
\tilde{u}=H v+w+E s \\
L^{*} \ni \tilde{u} \perp r \in L,
\end{array}\right.
$$

where $s \in \mathbb{R}^{n_{I}}$ is considered as a (fixed) parameter, to be adjusted so as to recover the full (3.13). The motivation for isolating these last three constraints is that they turn out to be optimality conditions of an optimization problem (parametrized by $s$ ) with good theoretical properties; this is the subject of the next section.

\subsection{Fixed-point formulation}

This section is devoted to the conic optimization problem

$$
\left\{\begin{array}{l}
\min \frac{1}{2} v^{\top} M v+f^{\top} v \\
H v+w+E s \in L^{*}
\end{array}\right.
$$

Its (quadratic) objective function

$$
J(v):=\frac{1}{2} v^{\top} M v+f^{\top} v
$$


is strongly convex and inf-compact (knowing that $M$ is assumed to be positive definite). Elementary arguments show that its feasible set

$$
\bar{C}(s):=\left\{v \in \mathbb{R}^{m}: H v+w+E s \in L^{*}\right\}
$$

is closed and convex. Thus, whenever $\bar{C}(s)$ is nonempty, (3.15) has a unique solution. This defines the mapping

$$
\mathbb{R}_{+}^{n_{I}} \ni s \mapsto v(s):=\underset{v \in \bar{C}(s)}{\operatorname{argmin}} J(v) \in \mathbb{R}^{m}
$$

which yields two more mappings, also well-defined:

$$
\mathbb{R}_{+}^{n_{I}} \ni s \mapsto\left\{\begin{array}{l}
\tilde{u}(s):=H v(s)+w+E s \in \mathbb{R}^{n d} \\
F(s):=\left(\left\|\tilde{u}_{T}^{1}(s)\right\|, \ldots,\left\|\tilde{u}_{T}^{n}(s)\right\|\right) \in \mathbb{R}^{n d}
\end{array}\right.
$$

The domain of these three mappings is the set of $s \in \mathbb{R}^{n_{I}}$ such that $\bar{C}(s) \neq \emptyset$.

Our motivation is that (3.14) makes up the optimality condition for (3.15) or (3.16), namely $-\nabla J(v) \in N_{\bar{C}(s)}(v)$. We proceed to make this more precise.

Lemma 3.2 For $v \in \bar{C}(s)$ (assumed nonempty), there holds

$$
H^{\top} N_{L^{*}}(H v+w+E s) \subset N_{\bar{C}(s)} .
$$

Proof. Introduce the notation $A v:=H v+w+E s$, so that the indicator function of $\bar{C}(s)$ is $\mathrm{i}_{\bar{C}(s)}=\mathrm{i}_{L^{*}} \circ A$, a convex function pre-composed by an affine mapping. Then use the chain rule for subdifferential calculus [HUL93, Thm XI.3.2.1]:

$$
H^{\top} \partial \mathrm{i}_{L^{*}}(A v) \subset \partial\left(\mathrm{i}_{L^{*}} \circ A\right)(v)=\partial \mathrm{i}_{C}(v) .
$$

In view of (3.6), this gives (3.20).

We can now proceed to relate our approach with the incremental problem.

Theorem 3.3 (Every complementary solution is a fixed point) Let $\left(v^{\star}, r^{\star}, \tilde{u}^{\star}, s^{\star}\right)$ solve the incremental problem (3.13). Then $v^{\star}=v\left(s^{\star}\right)$ and $F\left(s^{\star}\right)=s^{\star}$.

Proof. In view of (3.4), the complementarity relation in (3.13) says that $-r^{\star} \in N_{L^{*}}\left(\tilde{u}^{\star}\right)$, so $-H^{\top} r^{\star} \in H^{\top} N_{L^{*}}\left(\tilde{u}^{\star}\right)$. With (3.20), we conclude:

$$
-H^{\top} r^{\star}=-\left(M v^{\star}+f\right)=-\nabla J\left(v^{\star}\right) \in N_{L^{*}}\left(A v^{\star}\right) \subset N_{\bar{C}\left(s^{\star}\right)}\left(v^{\star}\right) .
$$

In other words, $v^{\star}$ satisfies the optimality conditions of (3.18) with $s=s^{\star}$ (see [HUL93, Thm VII.1.1.1] for example): $v^{\star}$ is the unique solution $v\left(s^{\star}\right)$ of this optimization problem. It follows that $\tilde{u}^{\star}=\tilde{u}\left(s^{\star}\right)$, and $F\left(s^{\star}\right)=s^{\star}$ of (3.19).

Note that $r^{\star}$ has no reason to be unique, unless $H^{\top}$ is injective.

To solve the incremental problem (3.13), we therefore have to find a fixed point of $F$. Now, a converse to the above theorem is desirable: will a fixed point of $F$ provide for sure a complementary solution? This relies upon (3.20) holding as an equality, which requires some subtle technicalities from convex analysis; we now turn to this question.

For all $i=1, \ldots, n$, set

$$
\mathcal{K}^{* i}=\left\{\begin{array}{cc}
\operatorname{int} K_{e^{i}, \frac{1}{\mu^{i}}} & \text { if } i \in I \\
K_{e^{i}, \infty} & \text { if } i \notin I
\end{array}\right.
$$

and introduce similarly to (3.12) and (3.17):

$$
\mathcal{L}^{*}=\mathcal{K}^{* 1} \times \cdots \times \mathcal{K}^{* n} \subset \mathbb{R}^{n d}
$$

(a convex cone included in $L^{*}$ ) and

$$
C(s):=\left\{v \in \mathbb{R}^{m}: H v+w+E s \in \mathcal{L}^{*}\right\}
$$


Remark 3.4 (Opening $L$ partially) Observe that $L$ is the product of two cones: $\prod_{i \in I} K^{i}$ (a non-polyhedral cone) and of $\prod_{i \notin I} K^{i}$ (an orthant of $\mathbb{R}^{n d}$, see (3.3)). To obtain $\mathcal{L}$ we open the first cone - replacing $\leqslant$ by $<$ in $(3.2)$; but we keep as is the second. Observe in particular that $\mathcal{L}$ is sandwiched between $L$ and the relative interior of $L$. This distinction in two cones is not strictly necessary for our development; but first, it is motivated by a companion work [CM10], which supplements our existence results of $\S 4$ below; second, it will allow us to put in perspective our result with some other's, see $\S 4.3$ below.

The point in introducing $C$ is that its nonemptiness guarantees optimality conditions for (3.15).

Lemma 3.5 (Qualification) For any s such that $C(s) \neq \emptyset$, the conic optimization problem (3.15) has a unique solution $v(s)$ and there exists $r$ such that $(v(s), \tilde{u}(s), r)$ solves (3.14).

Pro of. It is convenient for this proof to introduce the notation

$$
A:=\{(v, \tilde{u}): \tilde{u}=H v+w+E s\} \subset \mathbb{R}^{m} \times \mathbb{R}^{n d}
$$

and to formulate the conic optimization problem (3.15) as

$$
\min _{(v, \tilde{u}) \in \mathbb{R}^{m} \times \mathbb{R}^{n d}} Z(v, \tilde{u}), \quad \text { where } Z(v, \tilde{u}):=J(v)+\mathrm{i}_{A}(v, \tilde{u})+\sum_{i \in I} \mathrm{i}_{K^{i *}}\left(\tilde{u}^{i}\right)+\sum_{i \notin I} \mathrm{i}_{K^{i *}}\left(\tilde{u}^{i}\right) .
$$

Observe that $K^{i *}$ is a half-space of $\mathbb{R}^{d}$ when $i \notin I$ : the functions in the last sum above are all polyhedral.

Naturally, $\bar{C}(s) \supset C(s) \neq \emptyset$. Then ( $M$ is positive definite), the optimization problem has a unique solution $(v(s), \tilde{u}(s))$ characterized by the optimality condition $0 \in \partial Z(v(s), \tilde{u}(s))$.

Now the qualification assumption $C(s) \neq \emptyset$ is just what we need to subdifferentiate $Z$ using [Roc70, Thm. 23.8]: a subgradient of $Z$ is the sum of individual subgradients, each of which is easy to characterize. In fact, such a subgradient is an $m+n d$-vector of the form

$$
\left(\begin{array}{c}
M v+f \\
0
\end{array}\right)+\left(\begin{array}{c}
-H^{\top} r \\
r
\end{array}\right)+\sum_{i=1}^{n}\left(\begin{array}{c}
0 \\
q^{i}
\end{array}\right)
$$

where $r$ varies in $\mathbb{R}^{n d}$ and each $q^{i}$ is made up of $n d$-dimensional vectors, all zero except the $i^{\text {th }}$, which varies in $N_{K^{i *}}\left(\tilde{u}^{i}\right)$. Thus, the optimality condition $0 \in \partial Z(v(s), \tilde{u}(s))$ gives

$$
\begin{aligned}
& M v(s)+f=H^{\top} r \\
& r=-\sum_{i=1}^{n} q^{i}, \text { i.e. } r \in-N_{L^{*}}(\tilde{u}(s))=L \cap \tilde{u}(s)^{\perp}
\end{aligned}
$$

remembering that $(v(s), \tilde{u}(s))$ is feasible, we recognize (3.14).

Then the equivalence of the incremental problem (3.13) with $s=F(s)$ is easy to see:

Theorem 3.6 (Converse to Thm 3.3) Let $s^{\star} \in \mathbb{R}_{+}^{n_{I}}$ satisfy $F\left(s^{\star}\right)=s^{\star}$. In particular, $\bar{C}\left(s^{\star}\right) \neq \emptyset$, assume further that $C\left(s^{\star}\right) \neq \emptyset$. Then there exists $r^{\star}$ such that the associated $\left(v\left(s^{\star}\right), r^{\star}, \tilde{u}\left(s^{\star}\right), s^{\star}\right)$ solves (3.13).

Proof. Just apply Lemma 3.5: (3.14) holds for some $r^{\star}$ and the property $s^{\star}=F\left(s^{\star}\right)$ completes the system (3.13). The conclusion follows with Theorem 3.3.

Actually, the technical assumption $C(s) \neq \emptyset$ is useless in two dimensions:

Theorem 3.7 (Equivalence for $d=2$ ) Let the dimension be $d=2$. Then $\left(v^{\star}, r^{\star}, \tilde{u}^{\star}, s^{\star}\right)$ solves (3.13) if and only if $s^{\star}$ if a fixed point of $F$ and $v^{\star}=v\left(s^{\star}\right)$.

Proof. In fact, the conclusion of Lemma 3.5 holds even if $C\left(s^{\star}\right)$ is empty: when $d=2$, each $K^{i}-$ as well as each $K^{i *}$ - is a polyhedron, so the functions making up $Z$ are either finite-valued $(J)$ or polyhedral $\left(\mathrm{i}_{A}\right.$ and each $\left.\mathrm{i}_{K^{i *}}\right)$. 


\section{Existence of a solution to the incremental friction problem}

The previous section has provided a constructive way of solving (3.13): (i) using some internal subalgorithm to solve the optimization problem (3.15), (ii) compute a fixed point of $F(3.19)$, (iii) then we usually have a solution of the incremental friction problem (unless $C\left(s^{\star}\right)=\emptyset$, a rare event). This section studies the question whether such a fixed point exists.

It turns out that the assumption

$$
C(0) \neq \emptyset, \quad \Longleftrightarrow \quad \exists v \in \mathbb{R}^{m}: H v+w \in \mathcal{L}^{*} \quad \Longleftrightarrow \quad w \in \operatorname{Im} H+\mathcal{L}^{*}
$$

is relevant; in particular, it implies that each instance of (3.15) is well-posed (Lemma 4.2 below). This entails useful continuity properties of the mappings $C(\cdot)$ and $F(\cdot)$, which enable us to show existence via the Brouwer fixed-point theorem. We conclude the section with some comments concerning (4.1).

\subsection{Preliminary results}

First, $C(\cdot)$ enjoys a very handy property:

Lemma 4.1 (Monotonicity) The multifunction $C: \mathbb{R}^{n_{I}} \rightarrow \mathbb{R}^{m}$ of (3.22) is increasing; in other words for $s, t \in \mathbb{R}^{n_{I}}$ such that $s^{i} \leqslant t^{i}$ for all $i$, we have $C(s) \subset C(t)$. The same property holds for $\bar{C}(\cdot)$ of (3.17).

Proof. Let $s, t \in \mathbb{R}^{n_{I}}$ such that $s^{i} \leqslant t^{i}$ and take $v \in C(s)$, i.e. $(H v+w+E s)^{i} \in \mathcal{K}^{* i}$ for $i=1, \ldots, n$. Let us show component-wise that $H v+w+E t \in \mathcal{L}^{*}$.

We see from (3.9) that $(H v+w+E t)^{i} \in \mathcal{K}^{* i}$ if $i \notin I$. On the other hand, take $i \in I$; knowing that $e^{i} \in K_{e^{i}, \mu^{i}}$ and $\mu^{i}\left(t^{i}-s^{i}\right) \geqslant 0$,

$$
\mu^{i}\left(t^{i}-s^{i}\right) e^{i} \in K_{e^{i}, \mu^{i}}^{*}
$$

Add to $(H v+w+E s)^{i} \in \mathcal{K}^{* i}=\operatorname{int} K_{e^{i}, \mu^{i}}^{*}$ and invoke [HUL93, Lemma III.2.1.6]:

$$
z:=\frac{1}{2}(H v+w+E s)^{i}+\frac{1}{2} \mu^{i}\left(t^{i}-s^{i}\right) e^{i} \in \mathcal{K}^{* i}
$$

By positive homogeneity, $(H v+w+E t)^{i}=2 z$ also lies in $\mathcal{K}^{* i}$. Altogether, $H v+w+E t \in \mathcal{L}^{*}$.

The same argument can be applied to $\bar{C}$ - without calling for [HUL93, Lemma III.2.1.6].

Lemma 4.2 (Nonemptiness of $C$ ) Under Assumption (4.1), there is an open set $S$ containing $\mathbb{R}_{+}^{n_{I}}$ such that $C(s) \neq \emptyset$ for all $s \in S$. The mappings $v$ and $F$ are then well-defined on $S$.

Pro of. Consider the set of $s \in \mathbb{R}^{n_{I}}$ such that $C(s)$ is nonempty. By Lemma $4.1, \mathbb{R}_{+}^{n_{I}}$ is contained in it, since $C(0) \neq 0$. We proceed now to show that $\mathbb{R}^{n_{I}}$ is even contained in its interior, call it $S$.

In fact, take $s \in \mathbb{R}_{+}^{n_{I}}$ and $v \in \mathbb{R}^{m}$ such that $(H v+w+E s)^{i} \in \mathcal{K}^{* i}$ for $i=1, \ldots, n$. Then we see from (3.9) that, for some $\delta>0$,

$$
(H v+w+E t)^{i} \in \begin{cases}K_{e^{i}, \infty} & \text { for } i \in I \text { and all } t\left(\operatorname{then}(E t)^{i}=(E s)^{i}\right) \\ \operatorname{int} K_{e^{i}, \mu^{i}}^{*} & \text { for } i \in I \text { and }\left|t^{i}-s^{i}\right| \leqslant \delta .\end{cases}
$$

Thus, taking $t$ close enough to $s$ implies $H v+w+E t \in \mathcal{L}^{*}$, i.e. $v \in C(t)$ : our $s$ does lie in $S$.

The rest is Lemma 3.5 .

\subsection{Applying Brouwer's theorem}

To apply Brouwer's theorem, we need to prove that the mapping $F$ is bounded and continuous. We recall that a strongly convex function such as $J$ of (3.16) has bounded sublevel sets:

For given $t \in \mathbb{R}$, the sets $\left\{v \in \mathbb{R}^{m}: J(v) \leqslant t\right\}$ are bounded .

Lemma 4.3 (Boundedness) Under Assumption (4.1), the functions $v$ of (3.18) and $F$ of (3.19) are bounded on $\mathbb{R}_{+}^{n}:$ for some $R$,

$$
\|v(s)\| \leqslant R \text { and } \| F\left(s \| \leqslant R, \quad \text { for all } s \in \mathbb{R}_{+}^{n_{I}} .\right.
$$


Proof. From Lemma 4.2, $\bar{C}(s) \supset \bar{C}(0) \neq \emptyset$ for all $s \geqslant 0$. Fix $\bar{v} \in \bar{C}(0)$; then $J(v(s)) \leqslant J(\bar{v})<+\infty$ so that, by (4.2), $v(s)$ remains bounded when $s$ describes $\mathbb{R}_{+}^{n_{I}}$. Then observe from (3.1) that $\tilde{u}_{T}^{i}=(H v+w)_{T}^{i}$ for each $i$; hence $\tilde{u}$ is bounded as well, and so is $F$.

To prove continuity, we introduce the optimal value of (3.15), considered as a function of $s$ :

$$
\mathbb{R}^{n_{I}} \ni s \mapsto q(s):=J(v(s))=\min _{v \in \bar{C}(s)} J(v) \in \mathbb{R} \cup\{+\infty\},
$$

setting $q(s)=+\infty$ if $\bar{C}(s)=\emptyset$.

Proposition 4.4 (Convexity and continuity of the optimal value) The value-function $q$ of (4.3) is convex over $\mathbb{R}^{n_{I}}$. Under Assumption (4.1), it is continuous on $\mathbb{R}_{+}^{n_{I}}$.

Proof. For this proof, we consider the subset of $\mathbb{R}^{n_{I}} \times \mathbb{R}$ defined by

$$
\Gamma:=\left\{(s, t) \in \mathbb{R}^{n_{I}} \times \mathbb{R}: \exists v \in \bar{C}(s) \text { such that } J(v) \leqslant t\right\} .
$$

We claim that $\Gamma$ is closed and convex. To prove convexity, take $\left(s_{1}, t_{1}\right)$ and $\left(s_{2}, t_{2}\right)$ in $\Gamma$, with associated $v_{1}, v_{2}$ satisfying the above property. For $\alpha \in] 0,1\left[\right.$ and $v_{\alpha}:=\alpha v_{1}+(1-\alpha) v_{2}$, we directly see from convexity of $L^{*}$ and $J$ that

$$
v_{\alpha} \in \bar{C}\left(\alpha s_{1}+(1-\alpha) s_{2}\right) \text { and } J\left(v_{\alpha}\right) \leqslant \alpha t_{1}+(1-\alpha) t_{2},
$$

so that $\alpha\left(s_{1}, t_{1}\right)+(1-\alpha)\left(s_{2}, t_{2}\right)$ still lies in $\Gamma$.

To prove closedness, take a sequence $\left(s_{k}, t_{k}\right)_{k} \in \Gamma$ converging to some $(\bar{s}, \bar{t}) \in \mathbb{R}^{n_{I}} \times \mathbb{R}$, and an associated sequence $\left(v_{k}\right)_{k}$. For $k$ large enough, $J\left(v_{k}\right) \leqslant t_{k} \leqslant \bar{t}+1$ : the sequence $\left(v_{k}\right)_{k}$ is included in a sublevel set of $J$, and is therefore bounded. Extract a subsequence $\left(v_{k^{\prime}}\right)_{k^{\prime}}$ converging to $\bar{v} \in \mathbb{R}^{m}$ and pass to the limit for $k^{\prime} \rightarrow+\infty$ in

$$
H v_{k^{\prime}}+w+E s_{k^{\prime}} \in L^{*} \text { and } J\left(v_{k^{\prime}}\right) \leqslant t_{k^{\prime}} .
$$

In view of closedness of $L^{*}$ and continuity of $J$, the cluster point $(\bar{s}, \bar{t})$ lies in $\Gamma$ (associated to $\bar{v}$ ). In summary, $\Gamma$ is a closed convex set.

We observe now that $q(s)$ can be written (by pushing the objective function down to the constraints, with the help of an extra variable $t$ ) as

$$
q(s)=\min \{t:(s, t) \in \Gamma\} .
$$

Thus $q$ is the so-called lower-bound function of $\Gamma$; Theorem IV.1.3.1 of [HUL93] says that $q$ is convex (and lower semicontinuous) on $\mathbb{R}^{n_{I}}$. Besides, $q$ is finite-valued in a neighborhood of $\mathbb{R}_{+}^{n_{I}}$ (Lemma 4.2). It is therefore continuous on $\mathbb{R}_{+}^{n_{I}}$ by[HUL93, Prop. IV.2.1.2], which states that a convex function is continuous on the (relative) interior of its domain.

Proposition 4.5 (Continuity of the optimal solution) Under Assumption (4.1), v(.) of (3.18) is continuous on $\mathbb{R}_{+}^{n_{I}}$.

Proof. Fix $\bar{s} \in \mathbb{R}_{+}^{n_{I}}$ and let $s_{k} \rightarrow \bar{s}$; we have to show that $v\left(s_{k}\right) \rightarrow v(\bar{s})$. Extract from $\left(v\left(s_{k}\right)\right)_{k}$ (bounded, by Lemma 4.3) a subsequence $\left(v\left(s_{k^{\prime}}\right)\right)_{k^{\prime}}$ converging to some $\bar{v}$.

First, pass to the limit for $k^{\prime} \rightarrow \infty$ in the relation $H v\left(s_{k}\right)+w+E s_{k} \in L^{*}$. By continuity of $H$ and $E$ and closedness of $L^{*}$, we get that $H \bar{v}+w+E \bar{s} \in L^{*}$, i.e. $\bar{v}$ is feasible for (3.15) with parameter $\bar{s}$.

Besides, $J\left(v\left(s_{k^{\prime}}\right)\right) \rightarrow J(\bar{v})$ because $J$ is continuous; and $J\left(v\left(s_{k^{\prime}}\right)\right) \rightarrow J(v(\bar{s}))$ because $q$ is continuous (Proposition 4.4); hence $J(\bar{v})=J(v(\bar{s}))$. Thus, $\bar{v}$ is not only feasible but optimal. Because the optimal solution of (3.15) is unique, the arbitrary cluster point $\bar{v}$ has to be $v(\bar{s})$ itself.

We are now in a position to conclude.

Theorem 4.6 (Main result) Under Assumption (4.1), F has a fixed point $s^{\star}$ over $\mathbb{R}_{+}^{n_{I}}$, and there exists $r^{\star} \in \mathbb{R}^{n d}$ such that $\left(v\left(s^{\star}\right), r^{\star}, \tilde{u}\left(s^{\star}\right), s^{\star}\right)$ solves $(3.13)$.

Pro of. Because $v(\cdot)$ is continuous (Proposition 4.5), $\tilde{u}(\cdot)$ and $F(\cdot)$ of (3.19) are continuous.

Take $R$ of Lemma 4.3 and let $B \subset \mathbb{R}^{n_{I}}$ be the ball centered at 0 with radius $R$, intersected with $\mathbb{R}_{+}^{n_{I}}$. The restriction of $F$ to $B$ is continuous and maps $B$ (convex compact) into itself; it has a fixed point. Then apply Theorem 3.6. 


\subsection{Comments on the assumption}

The previous section has proved existence of a solution to the incremental problem, under the assumption that $C(0) \neq \emptyset$. A slightly more tolerant assumption would be

$$
\bar{C}(0) \neq \emptyset \text {, i.e. } \quad w \in \operatorname{Im} H+L^{*},
$$

which is mechanically more natural: it means that, at each contact point $i$, kinematics allow the relative velocity to lie in the dual $\left(K_{e^{i}, \mu^{i}}\right)^{*}$ of the friction cone. A property of this kind has to hold, indeed. For example, consider an over-simplified assumption:

$$
\exists v \in \mathbb{R}^{m}:(H v+w)_{N}^{i} \geqslant 0 \text { for } i=1, \ldots n,
$$

which just means that preventing penetration must be kinematically possible (unlike the meaningless situation of Figure 3 where the rigid ball is crushed between the motionless ground and a rigid plane with velocity $u_{0}$ ). Assumption (4.5) is

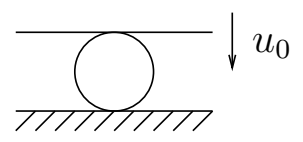

Fig. 3 Penetration cannot be prevented

(4.4), where all the friction coefficients $\mu^{i}$ are set to zero. As shown by Painlevé's example, it does not suffice to guarantee existence of a solution.

In [KP98], A. Klarbring and J.S. Pang use still another assumption: they formulate (2.3) as a quasi-variational inequality and prove existence if, in the notation of the present paper,

$$
\left[\begin{array}{c}
f \\
-w
\end{array}\right]^{\top}\left[\begin{array}{l}
v \\
r
\end{array}\right] \leqslant 0 \text { for all }(v, r) \text { satisfying }\left\{\begin{array}{l}
M v=H^{\top} r=0 \\
u=H v \\
\left(u^{i}, r^{i}\right) \in \mathcal{C}\left(e^{i}, \mu^{i}\right) \text { for all } i \in 1, \ldots, n
\end{array}\right.
$$

This turns out to slightly extend (4.4):

Proposition 4.7 If $M$ is positive definite, the above assumption means

$$
w^{\top} r \geqslant 0 \text { for all } r \in \operatorname{Ker}\left(H^{\top}\right) \cap L, \text { i.e. } \quad w \in\left(\operatorname{Ker}\left(H^{\top}\right) \cap L\right)^{*}=\operatorname{cl}\left(\operatorname{Im} H+L^{*}\right) .
$$

Proof. Let $T$ be (the closed convex cone) defined by

$$
M v=H^{\top} r=0, \quad u=H v, \quad\left(u^{i}, r^{i}\right) \in \mathcal{C}\left(e^{i}, \mu^{i}\right) \text { for all } i \in 1, \ldots, n .
$$

If $M$ is positive definite, the property $(v, r) \in T$ implies $v=0$ and $u=0$ : in this situation,

$$
(v, r) \in T \quad \Longleftrightarrow \quad v=0, H^{\top} r=0,\left(0, r^{i}\right) \in \mathcal{C}\left(e^{i}, \mu^{i}\right) \text { for all } i \in 1, \ldots, n .
$$

Examining (2.2) with $u=0$, the last conditions mean $r^{i} \in K^{i}$. Thus, the Klarbring-Pang assumption means

$$
w^{\top} r \geqslant 0 \text { for all } r \text { such that } H^{\top} r=0, r \in L .
$$

In other words, it means $w \in\left(\operatorname{Ker}\left(H^{\top}\right) \cap L\right)^{*}$. The result follows with [Roc70, Cor. 16.4.2], stating that the polar of an intersection is the closure of the sum of polars.

It goes without saying that closing a set enlarges this set: (4.6) is a more tolerant assumption than (4.4), which itself is more tolerant than (4.1). Our existence result is definitely weaker than that of [KP98] (especially as a positive semidefinite $M$ is accepted, there). This is the price to pay when replacing a complementarity problem by a minimization problem. Note also that Theorem 4.6 is slightly generalized in [CM10], where existence of a fixed point is proved under Assumption (4.4).

In fact,(4.1) could probably be weakened if we defined $v(s)$ as a solution of (3.14) instead of (3.15): the calculus rule necessary for Lemma 3.5 could then be avoided. However, this theoretical advantage would be fairly expensive for computational purpose: efficient algorithms abound to solve the nice minimization problem (3.15), whose solution behaves reasonably well when $s$ varies. The only equilibria (hence fixed points) not covered by our overly restrictive assumption 
(4.1) are those where Lemma 3.5 does not apply; to be sure, these solution should be fairly hard to compute. Indeed, the present work is more oriented toward constructive aspects.

The following synopsis summarizes the theoretical results available so far:

$$
\begin{array}{ccl}
w \in \operatorname{cl}\left(\operatorname{Im} H+L^{*}\right) & \Rightarrow & \text { existence for (2.3) via quasi-variational inequalities } \\
w \in \operatorname{Im} H+L^{*} & \Rightarrow & \text { existence of a fixed point, which may not solve (2.3) } \\
w \in \operatorname{Im} H+\mathcal{L}^{*} & \Rightarrow & \text { existence of a solution for (2.3), computable via optimization and fixed point. }
\end{array}
$$

Another aspect of the present work is the use of convex analysis, which appears as a handy tool to treat mechanical problems. For an illustration, we mention that the present material is actually extracted from [Cad09a], where the same results are also proved using convex analysis, but the arguments are different. For example, Lemma 3.5 is proved by invoking the dual of (3.15), namely

$$
\min _{r \in L} \frac{1}{2} r^{\top}\left(H M^{-1} H^{\top}\right) r+\left(w+E s-H M^{-1} f\right)^{\top} r .
$$

This alternative proof technique has its numerical advantage, as (4.7) might be easier to solve in some instances. Let us also outline the different proof techniques of Theorem 3.3 and Lemma 3.5; actually, both could have used the same calculus rule (either that of a sum, or that of a composition by an affine mapping). Convex analysis thus appears as a handy tool, but also versatile.

\section{Illustrations and discussion}

As far as existence of a solution to the incremental problem (3.13) is concerned, the previous sections have revealed three relevant assumptions: (4.1), (4.4), (4.6). They are indeed very close together, their difference being limited to theoretical hairsplit. In this section, we will focus on (4.4), which is the most natural and has a simple mechanical interpretation, mentioned at the beginning of $\S 4.3$. Our aim now is to mention a number of its practical applications: the frictionless case ( 5.1 ), Painlevé example ( $(5.2)$, and an important class of mechanical systems, where the external objects are motionless or in rigid-body motion ( $\S 5.6$ ). We also consider in $\S 5.7$ a situation where the assumptions hold for any $w \in \mathbb{R}^{n d}$.

\subsection{Frictionless case}

When all the friction coefficients are zero, the matrix $E$ is empty and the variables $s$ and $\tilde{u}$ disappear from (3.13). If $(v, u, r)$ is a solution, then in particular (4.5) is satisfied, so that the equivalent condition (4.4) is satisfied. In other words, (4.4) is actually a necessary and sufficient condition for existence in the frictionless case. Besides, there is no difference between (4.1), (4.4) and (4.6) because $L$ is polyhedral: each $K_{e^{i}, \mu^{i}}$ is a half-line.

Also from a numerical point of view, this case is much easier than the general case with actual friction: indeed, since there is no variable $s$, there is no fixed point problem either, it suffices to solve the convex minimization problem (3.15) once to get the solution. In addition, there is nothing nonlinear in this problem: (3.15) is a quadratic program with linear constraints. In a word: in the frictionless case, the incremental problem (3.13) reduces to one standard quadratic optimization problem.

\subsection{Painlevé's example I: necessity}

Recall Painlevé's Example 2.1 of $\S 2.2$, with $m=1, l=1, g=-1, h=1$ and $v_{0}=0$. We saw that a solution existed if and only if $u_{0} \leqslant 0$, or $u_{0}>0$ and $\tan \theta>\mu$. For this example, (4.4) means:

$$
\exists v \in \mathbb{R} \quad\left(u_{0}, 0\right)+(\cos \theta, \sin \theta) v \in K^{*} .
$$

Figure 4 shows the three possible situations, depending on the sign of $u_{0}$ and $\tan \theta-\mu$. It is clear from the picture that (4.4) is equivalent to the condition of Example 2.1: again it is necessary and sufficient.

\subsection{Painlevé's example II: non-necessity}

On the other hand, keeping the same data as in $\S 5.2$, just set $g=+1$ : gravity is now directed upwards. Also let $u_{0}=1$ and $h_{0}$ be such that $\theta<\arctan \mu$ when the contact is active. The same study as in Example 2.1 shows that a unique solution exists, the bar taking off under the effect of the (reversed) gravity. Yet the matrix $H$ and the vector $w$ are unchanged, so (4.4) does not hold: this condition is therefore not necessary in general. 


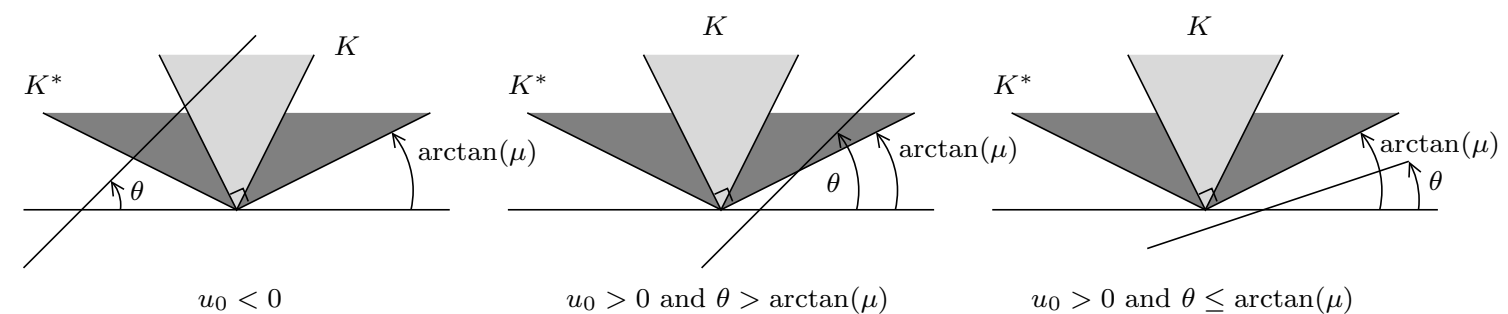

Fig. 4 Application of our criterion to the Painlevé example

\subsection{Painlevé's example III: non-uniqueness}

When rigid bodies are used, it is clear that contact forces are usually non-unique: because of the assumption of perfect rigidity, contact forces may compensate each other without any effect on the dynamics. For instance, for the static problem of a square lying on the ground, both solutions depicted on Figure 5 are valid (provided $\mu$ is large enough).
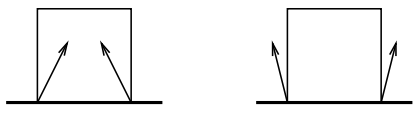

Fig. 5 A square lying on the ground

One may think that, although the forces are non-unique, the dynamic behaviour (that is to say, $v$ and $u$ ) should be unique. However, consider again Painlevé's example of $\S 5.3$ with $u_{0}=1, h_{0}=l / \sqrt{2}$ (so that $\theta=\pi / 4$ when the contact is active) and $\mu=2$. Again, we can solve the incremental problem by inspection, and we find that two outcomes are possible: either we apply no contact force and the bar takes off under the effect of the (reversed) gravity, or we apply a friction force which compensates gravity and yields a solution where the bar remains in contact and slides on the ground.

\subsection{Painlevé's example IV: some comments}

Intuitively, we would have expected the friction cone $L$ to appear in any reasonable criterion for existence. Painlevé's example shows why the appearance of the dual cone $L^{*}$ in Assumption (4.4) is actually natural: the property $H v+w \in L^{*}$ allows the friction force to act against penetration, by taking $u \in L^{*}$ and $r \in L$ so that $u^{\top} r \geqslant 0$ and the contact force tends to increase the velocity along the direction in which the force is applied. Otherwise, if this criterion is not met, then applying a contact force in a given direction can only decrease the velocity in that direction, which is counter-intuitive but may happen (it does, for example, when $u_{0}>0$ and $\mu>\tan \theta$ ).

Also note the difference between (4.1) and (4.4): when the former is met, it is possible to produce a (strictly) positive change of velocity in the direction of the applied contact force $r$ : one can get $u^{\top} r>0$. When only the latter is met, the situation is more ambiguous; one can only ensure $u^{\top} r \geqslant 0$, which means that the contact force produces only a nonnegative change of velocity in the direction $r$. One may think that this is not enough to ensure existence, but surprisingly [KP98] states the opposite, as it needs (4.6) and nothing more. This remark also indicates why (4.4) should be valid very often: unless the mechanical system is quite tricky, applying a force in a given direction usually produces motion in that same direction.

Section 5.3 explained why (4.4) is only sufficient in general: when the system is such that non-penetration occurs naturally without applying any contact force (say with the gravity directed upwards), there is no need to ensure that the contact force should act in the right direction! Requiring (4.4) here would be overly pessimistic.

Said otherwise, the criterion is purely kinematic and does not involve the mass matrix $M$ nor the term $f$ containing forces and initial conditions. As it does not use all the available information (in particular, it does not "know" whether contact forces are actually needed), its non-necessity is not surprising.

Along the same lines, note that (4.4) is of purely kinematic nature. As a result, it is intrinsic: although it is expressed in terms of $H$ and $w$, it actually depends only on the geometry of the system and not on the chosen parametrization.

\subsection{External objects with rigid motion}

The above-mentioned intrinsic character of (4.4) enables us to show that for a large class of systems, the incremental problem (2.3) always has a solution. Suppose that the external objects, if any, move as a single rigid body. Then, applying this same field of velocity to all the internal objects of the system yields zero relative velocity at all contact points (since 
the whole system is moving as a rigid object), which means that

$$
\exists v \in \mathbb{R}^{m}: H v+w=0 \in L^{*} ;
$$

this is exactly (4.4).

In particular, when there are no external objects or when the external objects are motionless, then $w=0$ in general (this is true for usual parametrizations, but may be false if one uses a time-dependent parametrization or a moving reference frame). In this case, taking $v=0$ suffices to obtain (4.4).

As an illustration, all systems depicted on Figure 6 have a solution to the incremental problem at each time-step. On this
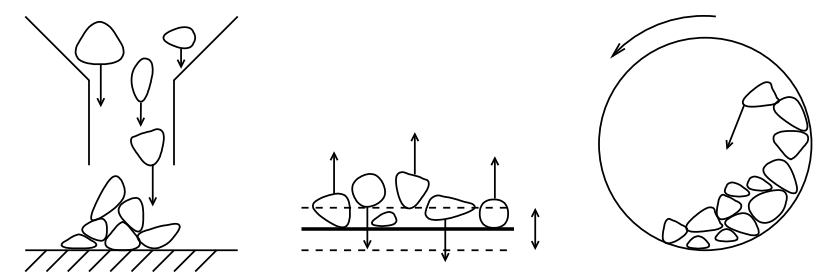

Fig. 6 Three classical situations where the criterion applies

figure, the first picture represents a very classical situation where (usually rigid) bodies fall from a funnel-shaped tank under gravity and pile on the ground, eventually producing a static stack. Since all external objects (the ground and the tank) are motionless, a solution always exists. The second picture represents an experiment where bodies are piled on a vertically vibrating plane under gravity; since the only external object (the plane) is moving as a rigid body, a solution always exists. The third example consists in a rotating drum filled with bodies; once again, the only external object (the drum) is moving as a rigid body and a solution exists.

By contrast, there are two external objects with imposed motion on Figure 2: the ground, which is fixed, and the upper end of the bar (point $A$ ) which moves with velocity $u_{0}$. They are of course required to allow non-existence.

\subsection{Conditions on $H$}

If the rows of $H$ are linearly independent, i.e. if $\operatorname{Ker}\left(H^{\top}\right)=\{0\}=(\operatorname{Im} H)^{\perp}$, then $\operatorname{Im} H$ is the whole of $\mathbb{R}^{n d}$, and so is $\operatorname{Im} H+w$. The intersection $\operatorname{Im} H+w \cap S$ is just $S$ for any set $S$, and assumptions (4.1), (4.4) and (4.6) are satisfied. No matter how $w$ is chosen in $\mathbb{R}^{n d}$, a solution exists to the incremental problem. Now the same property turns out to hold under a weaker assumption, namely

$$
\operatorname{Ker}\left(H^{\top}\right) \cap L=\{0\} \text {, }
$$

which is very relevant indeed. Among other things, distinguishing the three assumptions (4.1), (4.4) and (4.6) becomes irrelevant.

Theorem 5.1 The following two properties are equivalent:

(i) (5.1) holds;

(ii) for all $w \in \mathbb{R}^{\text {nd }}$, (4.1) holds - as well as (4.4) and (4.6).

When they hold, the incremental problem (3.13) has a solution for all $w \in \mathbb{R}^{\text {nd }}$.

Proof. Introduce the convex sets $G:=\operatorname{Im} H+L^{*}$ and $\mathcal{G}:=\operatorname{Im} H+\mathcal{L}^{*}$; recall Remark 3.4: $\mathcal{G}$ contains the relative interior of $G$.

Now (i) means $\left(\operatorname{Ker}\left(H^{\top}\right) \cap L\right)^{*}=\mathbb{R}^{n d}$; but from [Roc70, Cor. 16.4.2], this set is cl $G$ which is therefore the whole space; in particular, $G$ is full-dimensional. Knowing that the interior of a convex set and of its closure are the same ([HUL93, Prop. III.2.1.8]), $\mathcal{G}$ is full-dimensional as well and we write

$$
\mathcal{G} \supset \operatorname{int} G=\operatorname{int}(\operatorname{cl} G)=\operatorname{int} \mathbb{R}^{n d}=\mathbb{R}^{n d} .
$$

This exactly means (ii). The rest is Theorem 4.6.

Mechanical examples where the matrix $H$ has full row rank are usually found in the simulation of deformable bodies discretized by finite element techniques. In such an application, the number of degrees of freedom $m$ is usually far more larger than the number of reaction $n d$ at contact points. This fact is mainly explained by the role of the Coulomb friction 


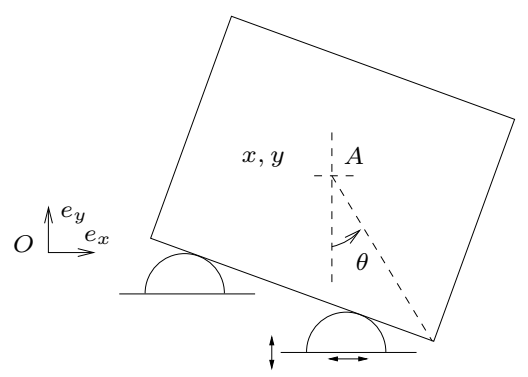

Fig. 7 A rigid rectangle on moving circular support.

which acts as a boundary condition, and then only on the degrees of freedom of the boundary. In such applications, the existence of solution is ensured as it has been already shown in [Jan81] and [Has83, Has84].

The condition (5.1) is illustrated on the simple example depicted on Figure 7, where a rectangle is supported on two circular bodies. The circle on the left is fixed and the right one is subjected to a rigid motion. In this case, The elements of the Kernel of $H^{T}$ are not in the product of the Coulomb cone. This situation is representative of many practical situations where a rigid body is subjected to many contacts. This situation results in existence of solutions but with non-uniqueness of the contact forces.

\section{Numerical Experiments}

Solving the incremental problem (2.3) via the approach suggested by $\S 3.3$ requires two nested algorithms. This section mentions some possibilities and presents some illustrations.

The outer algorithm must solve the fixed-point problem $s=F(s)$ coming from (3.19). One can use the standard method of successive approximations $s_{k+1}=F\left(s_{k}\right)$. It has the advantage of being simple but its convergence is not guaranteed; the main drawback is that the sequence $\left(s_{k}\right)$ may diverge. A sophisticated alternative is Newton's method, possibly coupled with a stabilizing device based on minimizing the least-square function $\|F(s)-s\|^{2}$; see [DS83]. However some theoretical work would be advisable to analyze the impact of the discontinuities in the derivatives of $F$. Other approaches are possible, consisting in applying nonsmooth optimization [Kiw85] to minimize some norm of $F(s)-s$, which is a so-called composite function [Fle87]. Here again, theoretical properties of the derivatives of $F$ need investigation.

To compute each $F(s)$, i.e. to solve the inner problem (3.15), several options are available.

(i) Directly use a solver which can handle a quadratic objective function and second-order cone constraints; such solvers exist and typically use interior-point methods.

(ii) Via the classical trick which pushes the quadratic objective function down to the constraints, reformulate the problem as a standard linear second-order cone program (SOCP); then use an off-the-shelf SOCP solver. We tried this approach, but the results were disappointing, in view of the significant increase in problem size due to the reformulation.

(iii) Replacing each constraint $\tilde{u}^{i} \in K^{* i}$ by its definition (3.2) results in an "ordinary" optimization problem with $n$ nonlinear constraints. Then any algorithm such as successive quadratic programming (SQP) can be used, see [Fle87]. If $n$ is really large, this may necessitate some customization of the quadratic program to be solved at each iteration.

(iv) As often done when solving friction problems, the second-order cones $K^{i}$ can be approximated by polyhedral ones [Kla86, AFSP91, PG96, ST96], in which case our subproblem (3.15) becomes an ordinary quadratic program. This can also be improved via a column generation technique, in which the number of facets approximating $L$ is dynamically managed along the process.

(v) Explicit formulae exist to project a point onto the second-order cone; one can therefore also use a naive projected gradient method to solve the dual problem (4.7). This is hardly an attractive option, given the notorious inefficiency of gradient methods, but it is easy to implement and gives a basis to compare more elaborate methods.

Having no solver (i) at hand, we now present some illustrations using a simple implementation: projected gradient (v), coupled with successive approximations for the fixed-point problem. We also present the faceting approach (iv), here with four facets. Note that we do not advocate faceted friction cones, since they introduce artificial anisotropy and do not seem likely to allow better computing times or robustness in our approach. We only report these results in order to get a rough idea of the improvement which could be gained by switching to an interior-point method in our context: in fact, we use OOQP [GW03] to solve the resulting quadratic program. 
We considered for our experimentations the very classical mechanical problem of simulating the dynamics of a granular material composed of rigid spheres, subject to gravity and to external unilateral constraints imposed by the ground and lateral walls. We simulated the complete dynamics, solving the incremental problem repeatedly. For all simulations, we used a time-step of 0.02 seconds. The friction coefficient we used depended on the experiment, but the restitution coefficient was always 0.2 . The tolerances were as follows:

- In the projected gradient algorithm, we checked if

$$
\frac{1}{n} \frac{\|\operatorname{Proj}(r-\nabla I(r))-r\|}{\|r\|+1} \leqslant 10^{-8}
$$

$I$ being the objective function in (4.7); besides, we allowed a maximum number of 250 iterations.

- For the faceting approach, we used the default settings of OOQP.

- The outer algorithm (the fixed-point loop) was stopped when

$$
\frac{1}{n} \frac{\|F(s)-s\|}{\|s\|+1} \leqslant \varepsilon
$$

with $\varepsilon=0.01$ by default. This criterion was met at all time-steps of all simulations, without reaching the allowed maximum number of 20 iterations (the largest number of subproblems to be solved for a given time-step was 12).

\begin{tabular}{c|c|c|c|c} 
\#beads & \#TS & \#contacts/TS & \#subprob./TS & CPU time/TS (ms) \\
\hline 150 & 352 & 239 & 3.3 & 768 \\
150 & 323 & 197 & 3.1 & 236 \\
300 & 308 & 506 & 3.2 & 1200 \\
300 & 272 & 393 & 3.5 & 1121 \\
600 & 300 & 741 & 3.3 & 1929 \\
600 & 255 & 524 & 3.5 & 949
\end{tabular}

Table 1 Overall results

Table 1 sums up the results. For three problems, with respectively 150, 300 and 600 rigid spheres (beads), it gives the statistics along the time-steps (TS), with projected gradient (first line) and interior points (second line); the friction coefficient is $\mu=0.3$ for all runs. It is remarkable that the number of inner problems to solve does not seem to increase with the problem size (knowing that the friction coefficient is fixed). Of course, the computing time does increase, since the time needed to solve each inner problem increases. Also, the computing time using projected gradient is not much worse than with interior points. This is partly due to the fact that warm restart is possible using projected gradient, but not with OOQP. This is not crucial at the beginning of the simulation, when the system is moving fast; in fact, interior points is by far the faster, then; but in the last steps of the simulation, when the stack of beads tends to stability, warm restarts become a huge advantage and projected gradient catches up.

Note that for a given problem (i.e. a given number of beads), the results depend on the solver. In particular, when the cone is faceted, the whole simulation tends to need less time-steps (and has therefore a lower average number of contacts per time-step, since this number increases during the simulation). For the same reason, the average computing time indicated in the last column of Table 1 should be considered with care.

Figure 8 shows the distribution of computing times in more detail during the simulation of the 600 beads problem. The influence of warm restarts is here obvious: the time needed by interior points increases more or less regularly with problem size (here indicated by the number of contacts), whereas projected gradient benefits a significant decrease for large problem sizes: this is due to the fact that larger numbers of contacts occur at the end of the simulation, when warm restarts are the most efficient.

In another experiment, we tested the influence of the friction coefficient. We considered the 300-bead problem and ran the simulation using projected gradient where the tolerance $\varepsilon$ in (6.1) was set to $10^{-4}$ and the maximum number of fixedpoint iterations was set to 50, so that convergence took longer and was more informative. We stopped the simulation at the first time-step where the number of contacts reached 250 and we plotted the value of the stopping criterion (6.1) during the fixed-point iterations. We repeated this experiment for $\mu$ varying from 0.1 to 0.9 , the results are reported in Figure 9 . The influence of the friction coefficient is clear: it changes the rate of convergence of the successive approximations algorithm. 


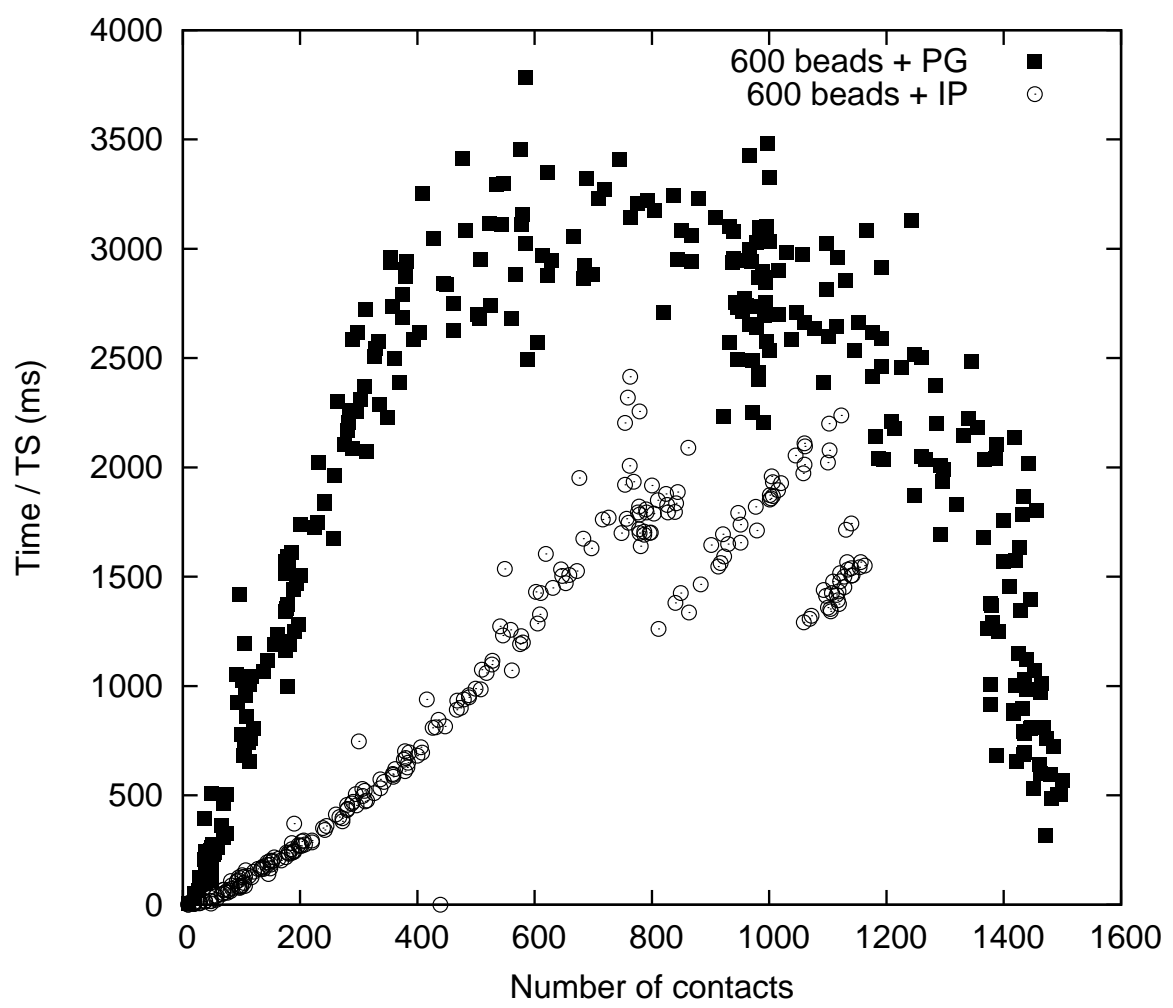

Fig. 8 Computing time per time-step as a function of the number of contacts

\section{Conclusion}

In this paper, we introduce a new formulation of the Coulomb friction problem in discrete time, as a fixed point problem. This allows us

- to prove existence of a solution using quite simple arguments,

- to tackle the problem numerically using an iterative procedure, which solves a sequence of convex quadratic problems under conic constraints.

Preliminary numerical experiments showed the practicality of the method. They revealed that the number of inner optimization problems to be solved is remarkably independent of the problem size, whereas it clearly increases with the value of the friction coefficient.

Possible directions for future work are to study the theoretical convergence of the method, and to investigate adequate algorithms to solve the inner optimization problem. Ideally, these algorithms should combine the efficiency of interior points and the ability to use warm restarts.

\section{References}

[AB08] V. Acary and B. Brogliato. Numerical Methods for Nonsmooth Dynamical Systems: Applications in Mechanics and Electronics, volume 35 of Lecture Notes in Applied and Computational Mechanics. Springer Verlag, 2008.

[Aba00] M. Abadie. Dynamic simulation of rigid bodies: Modelling of frictional contact. In B. Brogliato, editor, Impacts in Mechanical Systems: Analysis and Modelling, volume 551 of Lecture Notes in Physics (LNP), pages 61-144. Springer, 2000.

[AC91] P. Alart and A. Curnier. A mixed formulation for frictional contact problems prone to Newton like solution method. Computer Methods in Applied Mechanics and Engineering, 92(3):353-375, 1991.

[AC10] V. Acary and F. Cadoux. Applications of an existence result for the Coulomb friction problem. to appear in the edited volume of CMIS 2009 conference, Springer Verlag, 2010.

[AFSP91] A.M. Al-Fahed, G.E. Stavroulakis, and P.D. Panagiotopulos. Hard and soft fingered robot grippers. the linear complementarity approach. Zeitschrift für Angewandte Mathematik und Mechanik, 71:257-265, 1991.

[AG03] F. Alizadeh and D. Goldfarb. Second-order cone programming. Mathematical Programming, 95:3-51, 2003. 


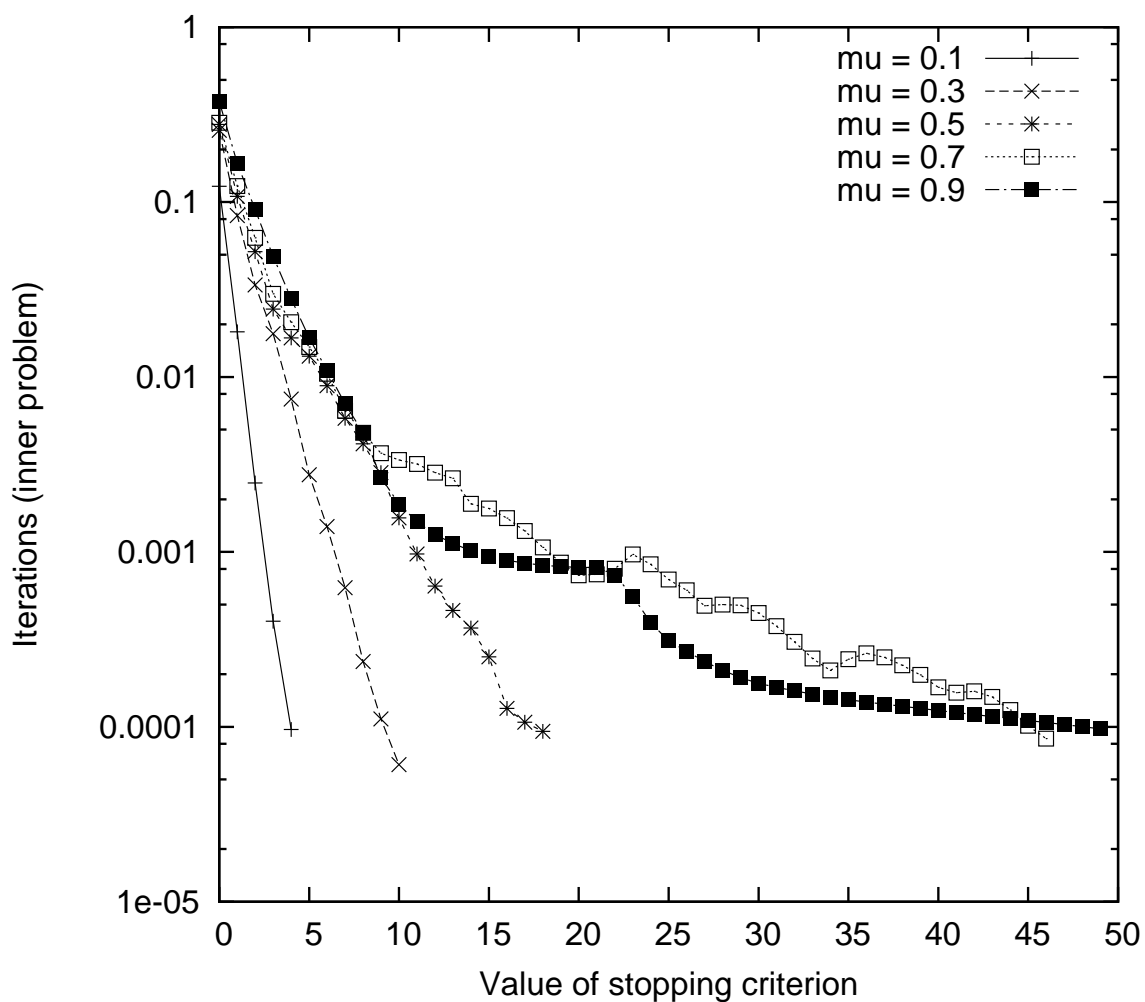

Fig. 9 Convergence $\log$ of the fixed point method for $\approx 200$ contacts

[Ala93] P. Alart. Injectivity and surjectivity criteria for certain mappings of $\mathbb{R}^{n}$ into itself; application to contact mechanics. (Critères d'injectivité et de surjectivité pour certaines applications de $\mathbb{R}^{n}$ dans lui-même; application à la mécanique du contact.). RAIRO, Modélisation Math. Anal. Numér., 27(2):203-222, 1993.

[Ala97] P. Alart. Méthode de newton généralisée en mécanique du contact. Journal de Mathématiques Pures et Appliquées, 76(1):83, 1997.

[And00] L.-E. Andersson. Existence results for quasi-static contact problems with Coulomb friction. Applied Mathematics and Optimization, 42:169-202, 2000.

[APS99] M. Anitescu, F. A. Potra, and D. E. Stewart. Time-stepping for the three dimensional rigid body dynamics. Computer Methods in Applied Mechanics and Engineering, 177:183-4197, 1999.

[Bal99] P. Ballard. A counter-example to uniqueness in quasi-static elastic contact problems with small friction. International Journal in Engineering Science, 37:163-178, 1999.

[Bal00] P. Ballard. The dynamics of discrete mechanical systems with perfect unilateral constraints. Archives for Rational Mechanics and Analysis, 154:199-274, 2000.

[Bar93] D. Baraff. Issues in computing contact forces for non penetrating rigid bodies. Algorithmica, 10:292-352, 1993.

[BB05] P. Ballard and S. Basseville. Existence and uniqueness for dynamical unilateral contact with Coulomb friction: a model problem. ESAIM, Math. Model. Numer. Anal., 39(1):59-77, 2005.

[BH09] M. Bostan and P. Hild. Weak formulations and solution multiplicity of equilibrium configurations with Coulomb friction. Math. Model. Nat. Phenom., 4(1):147-162, 2009.

[BV04] S. Boyd and L. Vandenberghe. Convex optimization. Cambridge University Press, 2004.

[CA88] A. Curnier and P. Alart. A generalized Newton method for contact problems with friction. Journal de Mcanique Thorique et Applique, supplément no 1 to 7:67-82, 1988.

[Cad09a] F. Cadoux. Optimisation et analyse convex pour la dynamique non régulière. PhD thesis, Univ. J. Fourier, Grenoble, 2009.

[Cad09b] F. Cadoux. An optimization-based algorithm for Coulomb's frictional contact. Esaim: Proceedings, 27:54-69, 2009.

[CKPS98] P. Christensen, A. Klarbring, J. Pang, and N. Stromberg. Formulation and comparison of algorithms for frictional contact problems. International Journal for Numerical Methods in Engineering, 42:145-172, 1998.

[CM10] F. Cadoux and J. Malick. Existence of a fixed point for a nonsmooth function arising in numerical mechanics. Nonlinear Analysis, 2010. submitted.

[DL72] G. Duvaut and J.L. Lions. Les Inéquations en Mécanique et en Physique. Dunod, Paris, 1972.

[DS83] J.E. Dennis and R.B. Schnabel. Numerical Methods for Unconstrained Optimization and Nonlinear Equations. PrenticeHall, Englewood Cliffs, 1983. 
[DSF91] G. De Saxcé and Z.-Q. Feng. New inequality and functional for contact with friction : The implicit standard material approach. Mech. Struct. \& Mach., 19(3):301-325, 1991.

[DSF98] G. De Saxcé and Z. Q. Feng. The bipotential method: a constructive approach to design the complete contact law with friction and improved numerical algorithms. Math. Comput. Modelling, 28(4-8):225-245, 1998.

[EJ03] C. Eck and J. Jarušek. Existence of solutions for the dynamical frictional contact problem of isotropic viscoelectic bodies. Nonlinear analysis, 53:157-181, 2003.

[EJK05] C. Eck, J. Jarušek, and M. Krbec. Unilateral Contact Problems: variational methods and existence theorems, volume 270 of Pure and Applied Mathematics. CRC Press, 2005.

[Fen95] Z.-Q. Feng. 2D and 3D frictional contact algorithms and applications in a large deformation context. Communications in Numerical Methods in Engineering, 11:409-416, 1995.

[Fle87] R. Fletcher. Practical Methods of Optimization. John Wiley \& Sons, Chichester (second edition), 1987.

[FM04] M. Frémond and F. Maceri, editors. Novel Approaches in Civil Engineering, volume 14 of Lecture Notes in Applied and Computational Mechanics. Springer Verlag, 2004.

[GB99] F. Génot and B. Brogliato. New results on painlv paradoxes. European Journal of Mechanics/A-Solids, 18:653-677, 1999.

[GCSL09] Ch. Glocker, E. Cataldi-Spinola, and R.I. Leine. Curve squealing of trains: Measurement, modelling and simulation. Journal of Sound and Vibration, 324(1-2):365-386, 2009.

[GW03] E.M. Gertz and S.J. Wright. Object-oriented software for quadratic programming. ACM Transactions on Mathematical Software, pages 58-81, 2003.

[Has83] J. Haslinger. Approximation of the signorini problem with friction, obeying the Coulomb law. Mathematical Methods in the Applied Sciences, 5:422-437, 1983.

[Has84] J. Haslinger. Least square method for solving contact problems with friction obeying Coulomb's law. Applications of mathematics, 29(3):212-224, 1984

[HS02] W. Han and M. Sofonea. Quasistatic contact problems in viscoelasticty and viscoplasticity. American Mathematical Society, International Press, 2002.

[HUL93] J.-B. Hiriart-Urruty and C. Lemaréchal. Convex Analysis and Minimization Algorithms. Springer Verlag, Heidelberg, 1993. Two volumes.

[JAJ98] F. Jourdan, P. Alart, and M. Jean. A Gauss Seidel like algorithm to solve frictional contact problems. Computer Methods in Applied Mechanics and Engineering, 155(1):31-47, 1998.

[Jan81] V. Janovský. Catastrophic features of Coulomb friction model. In J.R. Whiteman, editor, The Mathematics of Finite Elements and Applications, pages 259-264, 1981.

[Jar83] J. Jarušek. Contact problems with bounded friction. Coercive case. Czechosl. Math. J., 33:237-261, 1983.

[Jar84] J. Jarušek. Contact problems with bounded friction. Semioercive case. Czechosl. Math. J., 34:619-629, 1984.

[JE99] J. Jarušek and C. Eck. Dynamic contact problems with small Coulomb friction for visco-electic bodies. Existence of solutions. Math. Moels Methods Appl. Sci., 9(1):11-34, 1999.

[JM92] M. Jean and J.J. Moreau. Unilaterality and dry friction in the dynamics of rigid bodies collections. In A. Curnier, editor, Proc. of Contact Mech. Int. Symp., volume 1, pages 31-48. Presses Polytechniques et Universitaires Romandes, 1992.

[JT88] M. Jean and G. Touzot. Implementation of unilateral contact and dry friction in computer codes dealing with large deformations problems. J. Méc. Théor. Appl., 7(1):145-160, 1988.

[KB88] A. Klarbring and G. Björkman. A mathematical programming approach to contact problems with friction and varying contact surface. Computers \& Structures, 30(5):1185-1198, 1988.

[Kiw85] K.C. Kiwiel. Methods of Descent for Nondifferentiable Optimization. Lecture Notes in Mathematics 1133. Springer Verlag, 1985.

[Kla86] A. Klarbring. A mathematical programming approach to three-dimensional contact problems with friction. Computer Methods in Applied Mechanics and Engineering, 58:175-200, 1986. A. Klarbring. A mathematical programming approach to three-dimensional contact problems with friction. Computational Methods in Applied Mechanics and Engineering, 58:175200,1986 .

[KP98] A. Klarbring and J.S. Pang. Existence of solutions to discrete semicoercive frictional contact problems. SIAM Journal on Optimization, 8(2):414-442, 1998.

[LGW98] A.Y.T. Leung, C. Guoqing, and C. Wanji. Smoothing Newton method for solving two- and three-dimensional frictional contact problems. International Journal for Numerical Methods in Engineering, 41:1001-1027, 1998.

[MD87] E.N. Mitsopoulou and I.N. Doudoumis. A contribution to the analysis of unilateral contact problems with friction. Solid Mechanics Archives, 12(3):165-186, 1987.

[MD88] E.N. Mitsopoulou and I.N. Doudoumis. On the solution of the unilateral contact frictional problem for general static loading conditions. Computers \& Structures, 30(5):1111-1126, 1988.

[Mor66] J.J Moreau. Quadratic programming in mechanics: dynamics of one sided constraints. SIAM Journal on control, 4(1):153$158,1966$.

[Mor88] J.J. Moreau. Unilateral contact and dry friction in finite freedom dynamics. In J.J. Moreau and Panagiotopoulos P.D., editors, Nonsmooth Mechanics and Applications, number 302 in CISM, Courses and lectures, pages 1-82. CISM 302, Spinger Verlag, Wien- New York, 1988.

[Mor94] J.J. Moreau. Some numerical methods in multibody dynamics: Application to granular materials. European Journal of Mechanics/A-Solids, supp.(4):93-114, 1994.

[NJH80] J. Nečas, J. Jarušek, and J. Haslinger. On the solution of the variational inequality to the Signorini problem with small friction. Bollettino U.M.I., 5(17-B):796-811, 1980. 
[Pai95] P. Painlevé. Leçon sur le Frottement. Hermann, Paris, 1895.

[PG96] F. Pfeiffer and C. Glocker. Multibody Dynamics with Unilateral Contacts. Non-linear Dynamics. John Wiley \& Sons, 1996.

[PK94] J.K. Park and B.M. Kwak. Three dimensional frictional contact analysis using the homotopy method. Journal of Applied Mechanics, Transactions of A.S.M.E, 61:703-709, 1994.

[PS99] J.S. Pang and D.E. Stewart. A unified approach to frictional contact problem. International Journal of Engineering Science, 37:1747-1768, 1999.

[PT96] J.S. Pang and J.C. Trinkle. Complementarity formulations and existence of solutions of dynamic multi-rigid-body contact problems with Coulomb friction. Mathematical Programming, 73:199-226, 1996.

[RC01] R. Rocca and M. Cocu. Existence and approximation of a solution to quasistatic signorini problem with local friction. Int. J, Engrg. Sci., 39:1233-1255, 2001.

[RJMR96] F. Radjai, M. Jean, J.J. Moreau, and S. Roux. Force distributions in dense two-dimensional granular systems. Physical Review Letters, 77:274-277, 1996.

[Roc70] R.T. Rockafellar. Convex Analysis. Princeton University Press, 1970.

[ST96] D.E. Stewart and J.C. Trinkle. An implicit time-stepping scheme for rigid body dynamics with inelastic collisions and Coulomb friction. International Journal for Numerical Methods in Engineering, 39(15), 1996. reference tiree du site WILEY.

[Ste98] D. Stewart. Convergence of a Time-Stepping Scheme for Rigid-Body Dynamics and Resolution of Painlevé's Problem. Arch. Rational Mech. Anal., 154:215-260, 1998.

$\left[\mathrm{TLG}^{+}\right.$08] A.A. Transeth, R.I. Leine, Ch. Glocker, K.Y. Pettersen, and P. Liljebäck. Snake robot obstacle-aided locomotion: Modeling, simulations, and experiments. IEEE Transactions on Robotics, 24(1):88-104, 2008.

[WL08] P. Wriggers and T.A. Laursen, editors. Computational Contact Mechanics, volume 498 of CISM Courses and Lectures. Springer Verlag, 2008.

[Wri06] P. Wriggers. Computational Contact Mechanics. Springer Verlag, 2nd edition, 2006.

[ZVJL00] A. Zervos, I. Vardoulakis, M. Jean, and P. Lerat. Numerical investigation of granular interfaces kinematics. Mechanics of Cohesive-Frictional Materials, 5(4):305-324, 2000. 